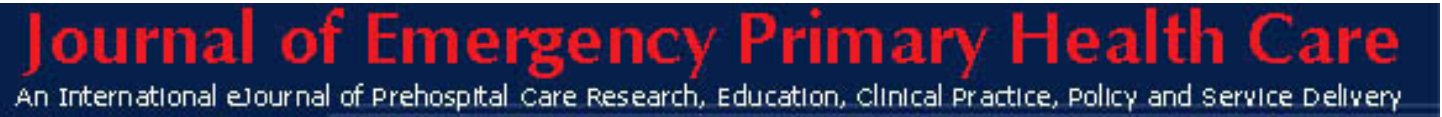

ISSN 1447-4999

\title{
COURSE REVIEW
}

\section{Cochrane Reviewers' Workshop}

Article 990128

\section{Leanne Sheen}

\section{Keywords}

Cochrane Collaboration; courses; literature reviews; meta-analyses; reviews; systematic reviews

\section{BACKGROUND}

The Cochrane Collaboration, established in 1993, is an international, non-profit, independent organisation, established to ensure that up-to-date information about the effects of healthcare interventions is readily available worldwide. ${ }^{1}$ It produces and disseminates systematic reviews of healthcare interventions, and promotes the search for evidence in the form of controlled trials of the effects of interventions. The Collaboration aims to introduce scientific rigour to minimise bias into the systematic review process by utilising an explicit and reproducible methodology. In conjunction with this, the reviews are updated regularly with the latest scientific evidence. Core funding is provided by royalties from its publishers, John Wiley and Sons Limited, which comes from sales of subscriptions to the Cochrane Library.

The introductory workshop for Cochrane Reviewers is a two day course designed to introduce researchers to foundation level information related to performing Cochrane reviews. The target audience are reviewers who have already identified a question of interest for review and are working on, or planning to start work on, a protocol.

\section{COURSE TEXT}

There is some required reading prior to commencement of the course. ${ }^{2-4}$ These articles provide a background of the rationale for conducting systematic reviews; the complexities involved in assessing the quality of included studies, and an introduction to the statistical methods used in Cochrane reviews. This allows course participants to become familiar with the materials and language that is used during the workshop. The Cochrane Collaboration also has its own supplementary software for processing Cochrane Reviews called `RevMan’ (Review Manager). Instructions for downloading this software are provided to participants with the pre-reading material. A folder with the course notes is available on registration. ${ }^{\mathbf{5 , 6}}$ Links to relevant websites with additional resources are made explicit throughout the course. These include links to The Cochrane Reviewers Handbook and Open Learning Materials. 


\section{WORKSHOP DETAILS}

The two day course in Melbourne was run by the Australasian Cochrane Centre who conduct courses approximately once per year in each state in Australia. The introductory workshop takes approximately 16 hours in total and is facilitated by experts in the field.

The number of participants enrolled in each course is limited, as small group activities and discussions are designed to enable collaboration of topic definition and development of master skills. The overall theme for the first day is 'Developing a Protocol for a Systematic Review'. Each participant begins the course by introducing themselves and providing a background of their research skills and activities. This information is then utilised in the small group activities so that all participants leave the course with further information relating to their individual reviews. Throughout the day, lectures are applied to a hypothetical review that is conducted and written by the group participants for the duration of the course. This application of theory to practice is an effective method of enhancing understanding and interest. In addition to the group work, the lecture content for the first day includes:

- Rationale and format of a protocol for Cochrane systematic review

- Formulation of the background and the question

- Locating and selecting appropriate studies

- Appraising trial quality

- Planning the methods of the review

- Useful information and resources

The second day is called 'Introduction to Analysis', where participants are provided with the analytical skills necessary for performing systematic reviews. The group hypothetical review is again used to demonstrate practical application throughout the day. Group work is utilised to perform meta-analysis interpretations and data extraction exercises. The pre-reading information is particularly useful in providing and supporting foundation knowledge for the second day content. In addition to the group work, the lecture content for the second day includes:

- Introduction to meta-analysis and forest plots

- Summary statistics for binary data

- Meta-analysis of continuous data

- Publication and related biases

- Heterogeneity

\section{DISCUSSION}

Overall, the course is an excellent product, suitable to anyone interested in, or practicing research and literature reviews. The course and its associated terminology may appear daunting for the novice researcher, however the actual structure is very supportive. The opportunity to network with other researchers from a variety of backgrounds and experience levels is invaluable. The target audience criteria is a little too rigid on the first day, however the information provided in relation to performing literature reviews, would be of great value to anyone undertaking post graduate research. Even if you are not interested in actually doing a systematic review, the course will provide helpful information to assist in conducting a rigorous literature review and critically analysing articles. 
The current guidelines for a Cochrane review are very rigid, with most review groups only conducting systematic reviews of randomised controlled trials (RCT's). One of the problems facing health professionals working in the community or prehospital setting is the lack of randomised controlled trials of prehospital interventions. This is further highlighted by the lack of prehospital systematic reviews in the Cochrane Library. It is difficult to conduct RCT's in the prehospital environment due to its uncontrollable and unpredictable nature, which in turn makes it difficult to systematically review prehospital evidence. Nevertheless, systematically reviewing the prehospital evidence that is available is vital in increasing the evidence base for prehospital care.

Interest is gathering to include protocols for reviewing qualitative research within Cochrane. ${ }^{7}$ Considering that the vast majority of literature pertaining to the prehospital or community setting is qualitative in nature, this is a vital step towards ensuring dissemination of high quality research and evidence based interventions across the broader prehospital population.

\section{CONCLUSION}

The Australasian Cochrane Centre facilitates a two day workshop to introduce researchers to the Cochrane Systematic Review process. Areas of the course have a generic content that is applicable to a variety of research methodologies, however some content is exclusively related to quantitative research and as such limits its applicability to reviews of interventions by randomised controlled trials. Further information on the Cochrane Collaboration can be found at: www.cochrane.org and www.cochranepehf.org

\section{RECOMMENDATION}

Consider attending this course prior to commencing a literature review at a postgraduate level. Inclusion of qualitative methodologies into the review process will highlight the usefulness of this valuable course. 


\section{References}

1. Cochrane C. Cochrane Collaboration Newcomers' Guide. In; 2005.

2. Juni P, Altman D, M E. Systematic reviews in health care: Assessing the quality of controlled clinical trials. BMJ 2001;323:42-46.

3. Lewis S, Clarke M. Forest plots:trying to see the wood for the trees. BMJ 2001;322:1479-1480.

4. Mulrow C. Systematic Reviews: Rationale for systematic reviews. BMJ 1994;309:597-599.

5. O'Connor D, Hedrick S. Developing a protocol for systematic review. In. Melbourne, Australia: Australasian Cochrane Centre; 2005.

6. O'Connor D, Hedrick S. Introduction to analysis. In. Melbourne, Australia: Australasian Cochrane Centre; 2005.

7. Vermeire E, Royen P, Peremans L, Hendrickx K, Coenen S, Griffiths F. The critical appraisal of focus group research articles. In: Cochrane Colloquia; 2000; Capetown: Cochrane Collaboration; 2005. 